\title{
Integrated photonic systems based on transformation optics enabled gradient index devices
}

\author{
Qi Wu, Jeremiah P Turpin and Douglas H Werner
}

Integrated photonics is expected to play an increasingly important role in optical communications, imaging, computing and sensing with the promise for significant reduction in the cost and weight of these systems. Future advancement of this technology is critically dependent on an ability to develop compact and reliable optical components and facilitate their integration on a common substrate. Here we reveal, with the utility of the emerging transformation optics technique, that functional components composed of planar gradient index materials can be designed and readily integrated into photonic circuits. The unprecedented design flexibility of transformation optics allows for the creation of a number of novel devices, such as a light source collimator, waveguide adapters and a waveguide crossing, which have broad applications in integrated photonic chips and are compatible with current fabrication technology. Using the finite-difference time-domain method, we perform full-wave numerical simulations to demonstrate their superior optical performance and efficient integration with other components in an on-chip photonic system. These components only require spatially-varying dielectric materials with no magnetic properties, facilitating low-loss, broadband operation in an integrated photonic environment.

Light: Science \& Applications (2012) 1, e38; doi:10.1038/lsa.2012.38; published online 23 November 2012

Keywords: gradient index material; integrated photonic system; quasi-conformal mapping; transformation optics

\section{INTRODUCTION}

Transformation optics (TO) provides a systematic method to manipulate light propagation by exploiting spatial mappings and distributions of constituent materials. ${ }^{1,2}$ Based on the property that Maxwell's equations are invariant under coordinate transformations, TO represents a powerful new design tool in controlling the trajectory of light and creating novel devices, such as invisibility cloaks, ${ }^{3}$ field concentrators ${ }^{4}$ and perfect 'black hole' absorbers. ${ }^{5}$ The important class of embedded coordinate transformations ${ }^{6}$ stands out by allowing discontinuities along transformation media boundaries. This unique property has facilitated the development of some of the more practical, but nonetheless remarkable TO devices, including reflectionless beam bends and splitters, ${ }^{7}$ polarization rotators ${ }^{8}$ and various flat lenses. ${ }^{9-11}$ Despite the many innovative applications, materials designed using TO are generally complex, exhibiting significant anisotropy and spatial dependence. $^{12,13}$ Recent progress on metamaterials promises a pathway for constructing TO devices; however, the associated absorption loss and limited bandwidth remain as major challenges for their practical application. One way to reduce material complexities is to employ quasi-conformal (QC) transformations, which can minimize the anisotropy of the constitutive materials, allowing all-dielectric implementations. ${ }^{14}$ As a result, nearly-isotropic gradient index (GRIN) materials with broad bandwidth and low losses can be employed, leading to functional QCTO devices, such as carpet cloaks and transformed Luneburg lenses in both the microwave and optical wavelength regimes. ${ }^{14-23}$

We apply embedded QC transformations towards the design of practical optical components for integrated photonic systems. There has been a growing interest in the development of more complex onchip systems by integrating miniaturized photonic devices of high functionality on a common substrate. ${ }^{24,25}$ Besides conventional devices with dielectric waveguides as key elements, engineered structures including photonic crystals and metamaterials can be employed to develop optical integrated devices or nanocircuits. ${ }^{26-28}$ Here, we introduce the concept of a new type of photonic integrated circuit, as shown in Figure 1, which consists of QCTO elements that can perform a variety of functions, such as light collimation, bending and coupling. Such TO-based GRIN components can effectively steer light throughout the entire volume of the device, providing compact implementation and a dramatic improvement in the control of light compared to conventional GRIN optics with simple axial, radial and spherical gradient profiles. ${ }^{29}$ Since the QCTO approach is inherently two-dimensional, it is felicitous and sufficient for the design of planar photonic devices which manipulate the spatial propagation of light within a thin layer. In contrast to general embedded TO designs, the QCTO components contain non-magnetic, dielectric-only materials. Therefore, they can be implemented with standard fabrication techniques, such as patterning of air holes or rods on a, e.g., silicon-on-insulator

The Pennsylvania State University, Department of Electrical Engineering, University Park, PA 16802, USA

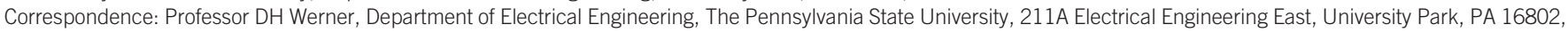
USA 


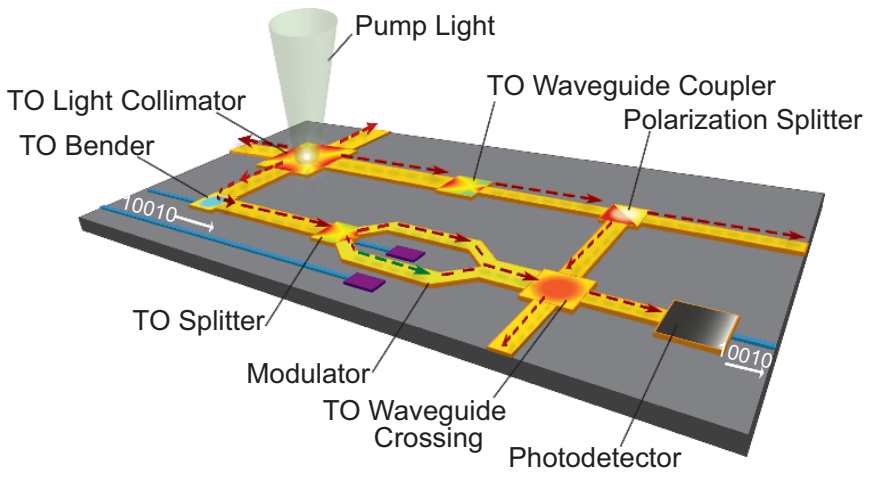

Figure 1 A schematic of a photonic integrated circuit composed of various TO components. A group of TO-based photonic components can be integrated with conventional optical and optoelectronic devices, including waveguides and photodetectors, on a common planar substrate. These TO building blocks create complex photonic devices and systems, which can provide a wide range of functionalities with applications in optical communications, information processing and sensing. The red arrows represent the propagation of light, which can be controlled by the TO components as well as on-chip electro-optic devices. TO, transformation optics.

platform, ${ }^{17-19,23}$ facilitating their application and integration into onchip photonic systems.

\section{MATERIALS AND METHODS}

To design functional devices such as light collimators, we consider coordinate transformations that can map a region containing a circle into a rectangle with flat surfaces. For instance, Figure 2a illustrates the geometry transformation from a circle with radius $R$ in the virtual space to a rectangle in the physical space. Two additional flat protrusions with a width of $w$ are chosen in order to facilitate orthogonal grid generation through QC mappings. In particular, the arcs on the top and bottom boundaries of the circular domain are mapped into two straight segments along the surfaces of the rectangular domain as shown by the solid red curves. When a point source is placed at the center of the virtual space in vacuum, the diverging cylindrical waves have equal-phase surfaces as concentric circles, including those curved

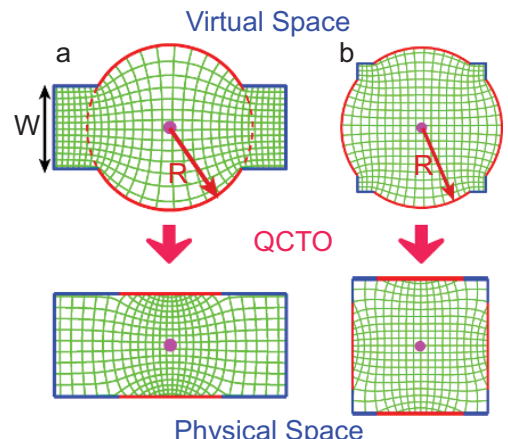

Figure 2 The QC transformations used to design integrated photonic components. (a, b) The QC mappings transform circular domains with protrusions in the virtual space into rectangular domains in the physical space. (a) The top and bottom surfaces of a circle are mapped into two straight segments along the surfaces of a rectangle (solid red). (b) Four arcs of a circle are mapped onto each side of a square (solid red). The resulting spatial grids and their inverse from the $\mathrm{QC}$ mappings are illustrated by green lines within the TO domains. A point source represented by a purple dot is embedded at the center of all the devices. QC, quasi-conformal; TO, transformation optics. boundaries in red. Since the circular arcs are mapped into straight lines in the new system, the emerging EM waves from these interfaces will have planar wave fronts, producing highly directive beams that can efficiently couple into neighboring components, such as optical waveguides. In Figure 2b, a QC transformation is performed over a circle with four corner-like protrusions to convert it into a square, where four equally distributed arcs are mapped in sequence onto the four sides of the square in the physical space. Consequently, an isotropically-radiating source at the center of the TO domain can be morphed into four highly collimated beams propagating in the outward normal direction to each face of the device.

The construction of transformation media for the TO designs in Figure 2 would normally require anisotropic materials with extreme parameters, ${ }^{10}$ which give rise to narrow bandwidths and high intrinsic losses arising from the resonant properties of inclusions. In the present work, QC transformations are employed to design TO devices composed of GRIN materials with minimal anisotropy. ${ }^{14,30}$ Such QC mappings are numerically generated orthonormal grids as shown in Figure 2, which can be implemented through a variety of different techniques. ${ }^{29-31}$ These mappings approximately satisfy the Cauchy-Riemann equations, and can be regarded as possessing all the properties of a conformal mapping for the purposes of the TO approach. ${ }^{32-34}$ Here, the QC mappings are obtained by solving Poisson's equation with NeumannDirichlet boundary conditions using the Poisson transform algorithm. ${ }^{30}$ As illustrated by Figure 2, the mesh grids created by the QC mappings possess strong orthogonality within the entire transformation domain, indicating that the anisotropy of the medium has been minimized.

The permittivity and permeability tensors of the transformation media can be found from the general TO design equations. ${ }^{13} \mathrm{We}$ assume a transverse electric polarized wave with electric field normal to the plane of propagation and characterize the TO devices by the spatial distribution of refractive index. As an example, Figure 3 shows the computed tensor elements of the refractive indices based on the transformation given in Figure 2a. The $x$ and $y$ axes indicate the normalized dimensions of the transformation domain in physical space, while the refractive index distributions are illustrated by the color maps used in the plots. It is found that the off-diagonal elements are
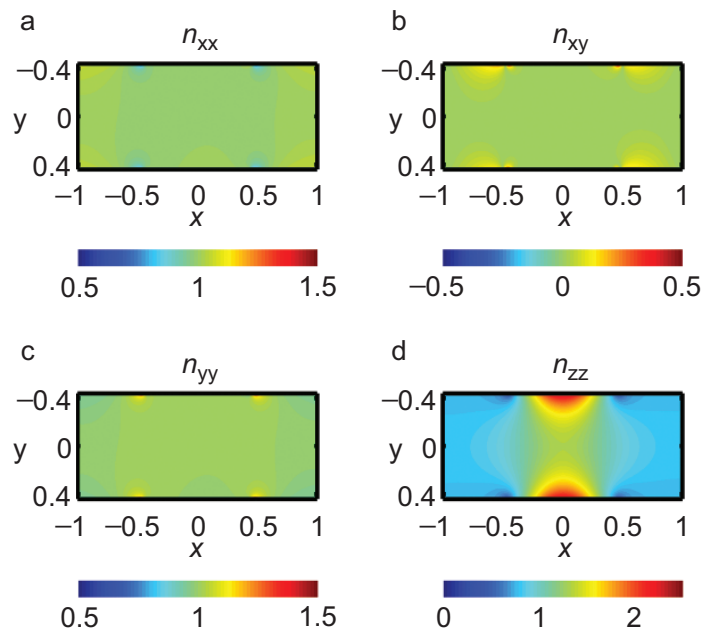

Figure 3 The QC transformations generate TO designs with an isotropic GRIN profile. The elements of refractive index tensor $\left(\mathbf{a}, n_{\mathrm{xx}}, \mathbf{b}, n_{\mathrm{xy}}, \mathbf{c}, n_{\mathrm{yy}}\right.$, and $\left.\mathbf{d}, n_{\mathrm{zz}}\right)$ for the QC transformation illustrated in Figure 2a. The interior region of the domain in the virtual space consists of free space. QC, quasi-conformal; GRIN, gradient index; TO, transformation optics. 
nearly zero due to the orthogonality of the grids, and all the in-plane diagonal elements are very close to unity except for $n_{\mathrm{zz}}$, which has a maximum value of 2.5. Therefore, the anisotropy of the transformation medium can be neglected, allowing TO devices to be created using only isotropic GRIN materials.

These types of TO designs composed of planar GRIN materials with two-dimensional index profiles can be implemented in slab waveguides, such as silicon-on-insulator wafers, leading to practical fabrication and integration with other photonic components in an on-chip system. ${ }^{23}$ For instance, structured dielectrics comprised of subwavelength holes etched in a waveguide can be used to create an effective medium with desired GRIN distributions. ${ }^{17}$ Using this technique, a refractive index ranging from the bulk value of the dielectric host medium to near unity is achievable under certain fabrication constraints, allowing for advanced QCTO designs with a large index variation. ${ }^{35}$ As a result, this method has been successfully employed for experimental demonstrations of several TO devices in the near infrared region. ${ }^{17-19,23,35}$ Alternatively, the GRIN distributions necessary for the QCTO devices can be implemented by controlling the thickness of the guiding layer of a slab waveguide. ${ }^{36,37}$ Since the effective mode index of the waveguide is dependent on the thickness of the guiding layer, the GRIN profile of a TO design can be translated into a height map of the guiding layer. Based on this method, low-loss Luneburg lenses with strong index variations have also been demonstrated. ${ }^{38,39}$

\section{RESULTS AND DISCUSSION}

We first design a square quad-beam collimator by following the QC mapping illustrated in Figure 2b. Based on the geometry transformation, waves that emerge from each face of the device possess planar equal-phase surfaces, which produce collimated light beams normal to the surfaces. ${ }^{40-43}$ When connected to silicon waveguides at its collimating surfaces, this TO component efficiently couples light emitted from a point source at the center of the device into each waveguide. By following the general TO design equations, ${ }^{13}$ the required effective parameters of the transformation medium are calculated. As shown in Figure 4a, the GRIN profile of the collimator exhibits four-fold symmetry due to the transformation geometry and has a high index at each collimating surface. The highest refractive index found at the edge of the device can be adjusted by controlling the geometries and dimensions of the transformation domain. Therefore, the transformation is engineered such that the indices of the silicon waveguide and the QCTO element are mostly matched at the boundaries with an index of 3.4, leading to minimal reflection.

In order to characterize the performance of the TO collimator coupled with waveguides, full-wave numerical simulations are performed using the finite-difference time-domain (FDTD) method. Figure $4 \mathrm{~b}$ demonstrates the light intensity distribution excited by a point source with a wavelength of $1.5 \mu \mathrm{m}$, where the emitting light is smoothly guided inside the TO collimator before entering the connecting waveguides. Low reflections at the interface are evidenced as the interference fringes are fairly weak inside the TO collimator. In fact, FDTD simulations reveal that over $98 \%$ of the emitted energy is coupled into the waveguides through the TO collimator, demonstrating a high coupling efficiency and low insertion losses.

In addition to the light source collimator, the QC transformation technique can be employed to design many other GRIN components with versatile functionalities. To further demonstrate the flexibility of this design tool, we present several waveguide adapters that are critical components in integrated photonic systems as depicted in Figure 1. One example is a waveguide coupler that can connect different-sized a
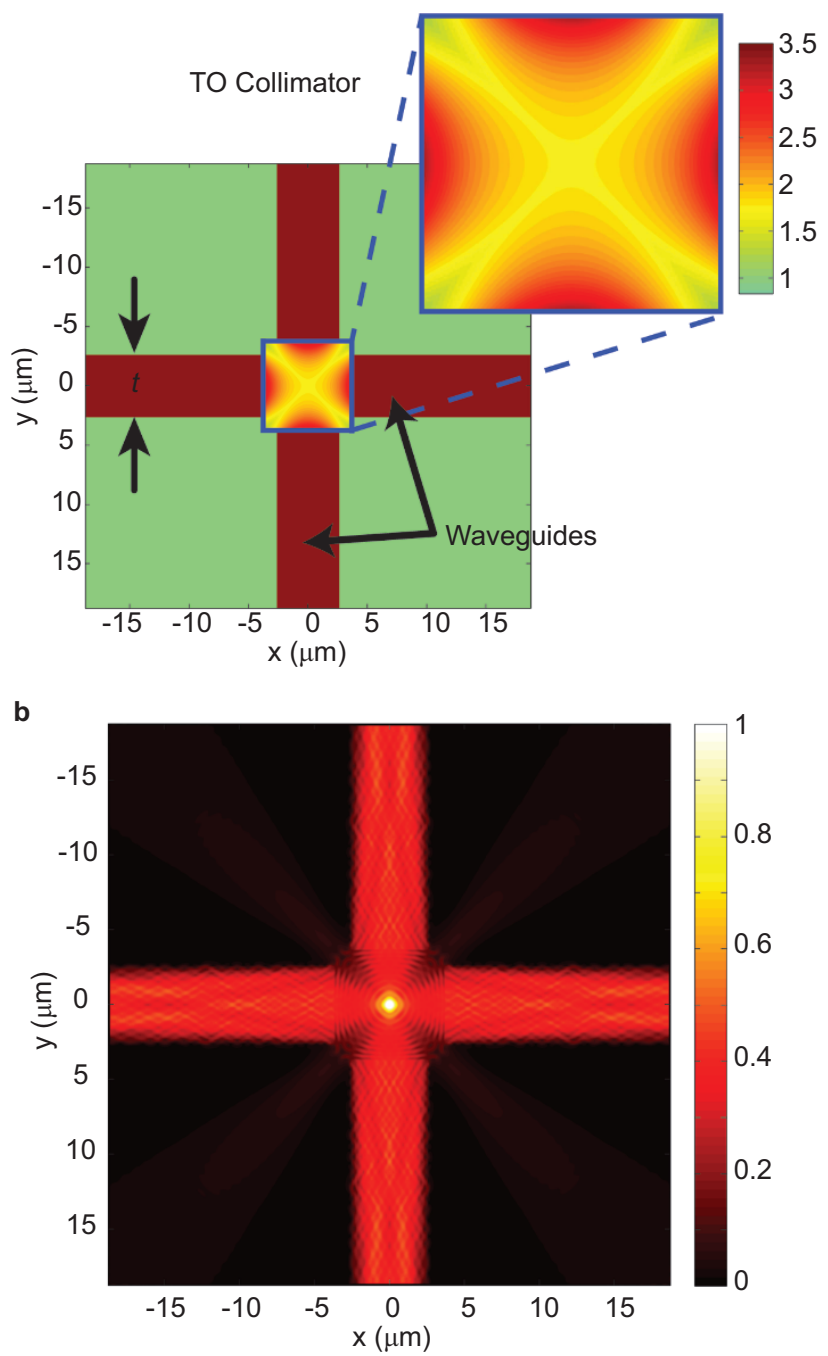

Figure 4 A quad-beam TO collimator connects four silicon waveguides. (a) The square quad-beam collimator measuring $7.5 \mu \mathrm{m}$ on a side connects four silicon waveguides with $t=5 \mu \mathrm{m}$. The inset shows the refractive index profile of the QCTO device. (b) The light intensity distribution excited by a point source at the center of the device demonstrates efficient coupling from the light source to all the waveguides. The wavelength of the light source is $1.5 \mu \mathrm{m}$. QC, quasi-conformal; TO, transformation optics.

optical waveguides by converting the guided modes. ${ }^{44}$ In contrast to a conventional coupler which requires a long adiabatically tapering section to reduce losses, the TO-based design approach leads to compact devices as the light propagation can be controlled effectively through the GRIN distribution over the entire transformation medium.

The coupler is comprised of two GRIN elements, as shown by the black dashed box in Figure 5a. The index distributions of both elements are obtained based on the QC mapping illustrated in Figure 2a. Due to the mirror symmetry of the refractive index profile in Figure 3d, each element of the waveguide coupler consists of half of the transformation medium. Furthermore, the geometrical transformations are chosen so that the resulting TO coupler matches the size and refractive index of the waveguides at both the input and output surfaces. In this way, light carried by the input waveguide from the left is compressed into a narrow beam by the first element of the coupler before experiencing a subsequent expansion to match the size of the 
a

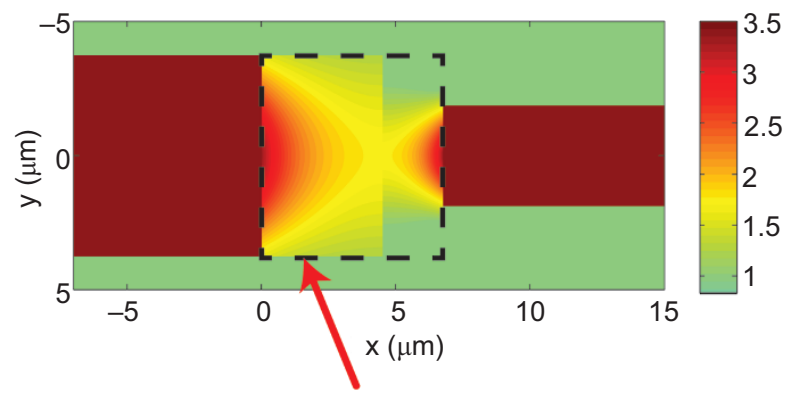

Waveguide Coupler
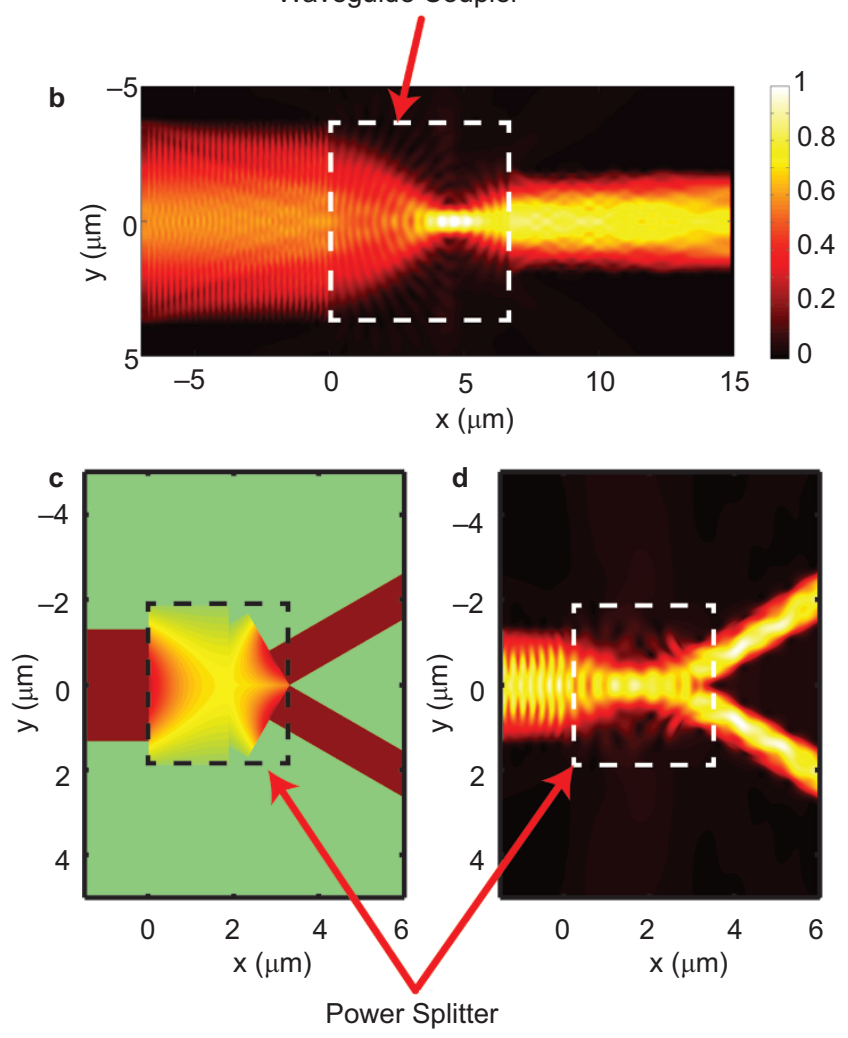

Figure 5 TO-based GRIN waveguide adapters. (a) A TO waveguide coupler connects two silicon waveguides with a width of $7.5 \mu \mathrm{m}$ and $3.75 \mu \mathrm{m}$, respectively. The coupler device, illustrated in the black dashed box, has a size of $7.5 \mu \mathrm{m} \times 6.7 \mu \mathrm{m}$. (b) Light intensity distribution obtained from FDTD simulations. Light from the input waveguide on the left couples well into the output waveguide through the QCTO component. (c) The refractive index profile of a QCTO power splitter which divides light from the input waveguide $(2.5 \mu \mathrm{m})$ between two output waveguides $(1.0 \mu \mathrm{m})$. The TO splitter within the dashed box has a footprint of $3.7 \mu \mathrm{m} \times 3.3 \mu \mathrm{m}$. (d) FDTD simulation demonstrates equal division of optical power between two output waveguides through the TO splitter. The wavelength of the light in both (b) and (d) is $1.5 \mu \mathrm{m}$. The color scales for (c) and (d) are identical to those in (a) and (b). FDTD, finite-difference time-domain; GRIN, gradient index; QC, quasi-conformal; TO, transformation optics.

output waveguide. Figure $5 \mathrm{~b}$ demonstrates the performance of the device, through which light is smoothly guided and coupled between two silicon waveguides of different sizes. Despite a small amount of reflective and scattered light, which are found to be $1.6 \%$ and $3.4 \%$, respectively, such a compact TO waveguide coupler provides a coupling efficiency with up to $95 \%$ transmission. Further numerical studies reveal that the TO coupler maintains a high transmission above $94 \%$ at wavelengths between $1.0 \mu \mathrm{m}$ and $2.0 \mu \mathrm{m}$, covering the entire infrared telecommunications band. Meanwhile, it is interesting to note that the incident light is focused in the middle of the coupler, producing an enhanced electric field that is five times stronger than that in the input waveguide. As a result, this TO component, together with the associated waveguides, provides an integrated platform that is suitable for investigating light interaction with optical media, such as active and nonlinear materials.

Another commonly used component in integrated photonics is a power splitter, which equally divides power from an input waveguide between two output waveguides. The Y branch and multimode interference element are often used; however, these devices have large footprints and exhibit limited bandwidth, respectively. ${ }^{24,25}$ Here, we combine properly engineered TO elements to form a new type of power splitter. As shown in Figure $5 c$, the TO version of the splitter is composed of three GRIN elements, each connecting to an optical waveguide. The GRIN profile of each element is found by performing the QC mapping illustrated in Figure 2a. Light from the input waveguide is divided between two output waveguides, which form a $60^{\circ}$ angle with respect to each other. Figure $5 \mathrm{~d}$ illustrates the light intensity distribution predicted by FDTD simulations. As we expect, the incident light gradually couples into the first element of the TOGRIN splitter before being separated into the two output waveguides by the second half of the device. Due to the matched indices at all the surfaces of the device, the optical power is efficiently delivered and equally distributed in the output waveguides. Moreover, such combined TO splitters can be easily adjusted to integrate with waveguides that have different sizes and orientations, making them promising candidates for compact, broadband integrated photonic systems.

Another essential component in integrated photonics is the waveguide crossing, which allows optical signals carried by waveguides to be routed and interconnected within a limited space, providing the possibility for high-density integration of optical devices in photonic circuits. ${ }^{24,25}$ In order to mitigate insertion loss and crosstalk at the intersections of optical waveguides, either a resonator or a mode expander type crossing device is needed. ${ }^{45,46}$ By applying an appropriate QCTO approach to engineer the GRIN profile of the device, we design a new type of waveguide crossing that can channel the light propagation throughout the transformation medium without any wave guiding structures. Therefore, compact TO-based crossing devices can be realized to operate over a wide bandwidth. TO devices that can bend and shift light beams have been proposed and demonstrated through both numerical simulations and experimental characterization. $^{10,23,32}$ Here, we investigate GRIN elements which can process two or more beams simultaneously, and demonstrate the crossing functionality by connecting the TO device with a set of input and output waveguides.

For the development of a waveguide crossing, we have utilized the geometrical mapping illustrated in Figure 2a, in which a circular domain in the virtual space is transformed into a rectangular domain with flat surfaces in the physical space. Since light rays in both spaces must remain normal to the boundaries (solid red lines), the light will bend to pass through the origin in the physical space and exhibit a lateral shift when exiting the device. ${ }^{23}$ Instead of assuming a vacuum in the virtual space as in most of the TO designs, we assign a Maxwell fisheye lens with a radial GRIN distribution given in Equation (1) to the circular region as shown in Figure $6 a$, where $R$ is the radius of the circular region and $0 \leqslant r \leqslant R$.

$$
n(r)=\frac{2}{1+(r / R)^{2}}
$$


a
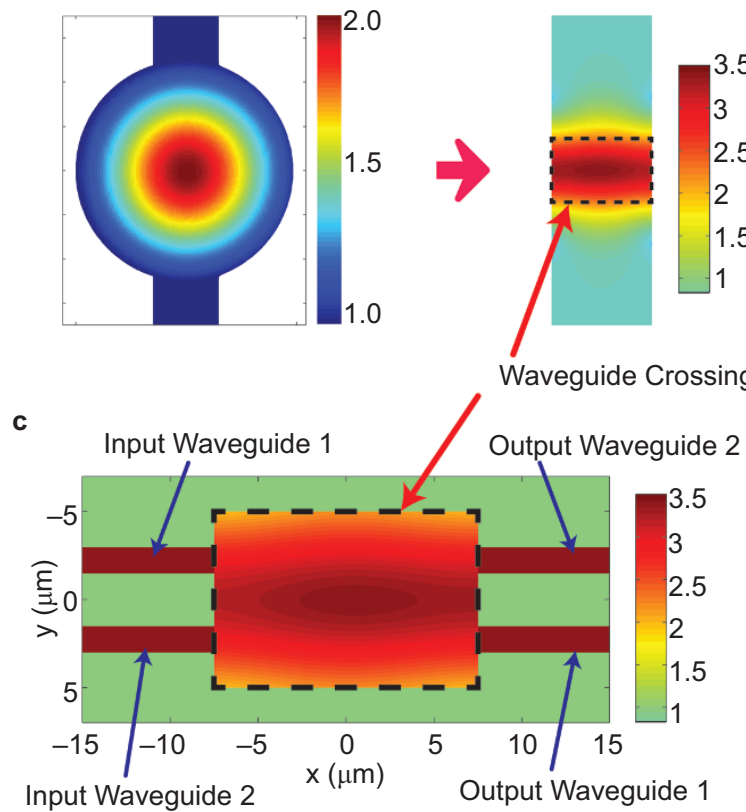

b

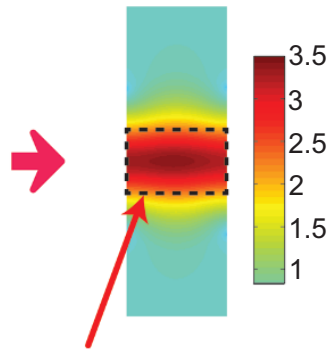

Output Waveguide 2
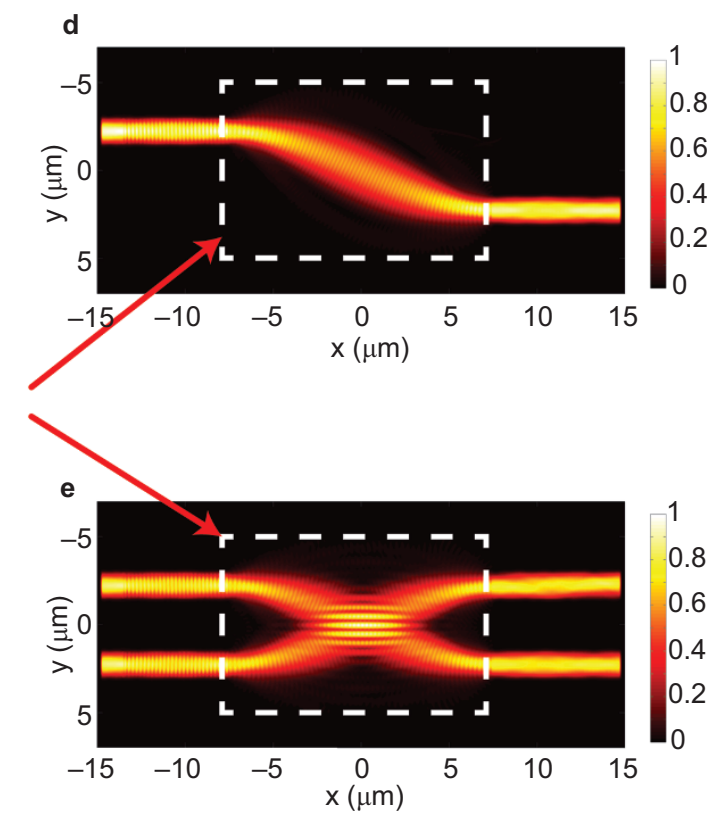

Figure 6 A TO-based GRIN waveguide crossing. (a) The refractive index profile of a Maxwell fisheye lens in a circular domain containing protrusions in the virtual space. (b) The GRIN profile of the transformed rectangular domain in the physical space. (c) A TO waveguide crossing connects two silicon waveguides with a width of $1.5 \mu \mathrm{m}$ on either side of the device. The crossing device, illustrated in the black dashed box, has a size of $15 \mu \mathrm{m} \times 10 \mu \mathrm{m}$. (d) Light intensity distribution obtained with input waveguide 1 illuminated. Light from the input waveguide bends down and couples well into the output waveguide 1 through the QCTO component. (e) Light intensity distribution obtained with both input waveguides 1 and 2 illuminated. The wavelength of the light in both (d) and (e) is $1.5 \mu \mathrm{m}$. GRIN, gradient index; QC, quasi-conformal; TO, transformation optics.

Recent applications of the TO approach to conventional GRIN optics, such as the Luneburg lens and Maxwell fisheye lens, have led to devices with more advantageous functionalities. ${ }^{21,22,29}$ Analogously, it is found that the inherent focusing property of the Maxwell fisheye lens can efficiently suppress the diffraction of light in the TO crossing design and significantly reduce loss and crosstalk. The resulting GRIN profile of the transformed medium is shown in Figure $6 \mathrm{~b}$, where the center region within the dashed black box is truncated and employed as a crossing device.

To demonstrate the waveguide crossing behavior, the GRIN device is connected to two input and two output waveguides with reversed order on either side of the device as shown in Figure $6 \mathrm{c}$. The crossing device, illustrated by the black dashed box, has a size of $15 \mu \mathrm{m} \times 10 \mu \mathrm{m}$ and a refractive index ranging from 2.0 to 3.4. All the connecting $\mathrm{Si}$ waveguides possess a width of $1.5 \mu \mathrm{m}$, while the adjacent waveguides on each side of the device are separated by a distance of $3 \mu \mathrm{m}$. FDTD simulations were performed to illustrate the light shifting and crossing effects throughout the TO device. In Figure 6d, only the input waveguide 1 is illuminated. It is evident that light is smoothly guided and bended within the TO crossing device, which interconnects the two waveguides placed symmetrically about the center of the device. Due to the imaging property of the Maxwell fisheye lens, the diffraction of light is compensated as the wave is focused right in front of the output waveguide and the lateral expansion of the beam is well confined within the GRIN region. As a result, low insertion loss and crosstalk are realized with the numerical simulations predicting a transmission of $98 \%$. When both input waveguides are illuminated, as shown in Figure 6e, the TO device provides the desired crossing performance by routing two beams into the corresponding output waveguides in an inversed order. Although some interference fringes can be observed at the intersection area of the two beams, the two optical signals are guided independently and couple into the output waveguides with transmission of over $98 \%$ in each case. By increasing the size of the TO crossing element and including more waveguide ports, it is possible to further extend the number of optical signals across the device and thereby further enhance the integration density of neighboring devices. Moreover, the TO device demonstrated can also be employed as a directional coupler with broader bandwidth compared to conventional designs based on coupled modes. ${ }^{24}$

By exploiting the flexibility of the embedded QCTO approach, compact GRIN components such as the waveguide adapters and crossings have been realized and provide superior performance compared to their state-of-the-art counterparts. Furthermore, the TO components can introduce new functionalities to conventional GRIN optics and significantly improve the system level integration of photonic devices. For instance, the TO collimators, which effectively couple micro- and nano-scale photon sources (i.e., quantum dots) with photonic waveguides, provide a path to integrate on-chip light sources with high efficiency and thus broaden their range of applications. In fact, the embedded QCTO approach enables a class of all-dielectric GRIN components, such as beam shifters, ${ }^{23}$ benders ${ }^{32}$ and even polarization splitters, in which light with both polarizations can be independently controlled, ${ }^{47}$ which are promising for integrated photonic systems with enhanced functionalities and compact size. By utilizing the focusing and enhancement of light offered by some TO devices, integrated optical sensors and modulators with stronger light-matter interaction can also be realized. Moreover, the recent development of plasmon nano-lasers offers the possibility of active photonic circuits; however, their integration and application have been hindered due to the lack of collimated emission of laser light. ${ }^{48,49}$ In view of the recent interest in TO techniques for plasmonics, ${ }^{50-52}$ we envision that the approach and devices demonstrated here could provide an enabling technology for 
efficient coupling of nanoscopic light, leading to a new type of nanocircuit architecture based on controlling surface plasmons.

\section{CONCLUSIONS}

In summary, we have designed and demonstrated several TO-inspired GRIN components for integrated photonic systems using full-wave FDTD simulations. An embedded QC transformation approach is employed, yielding devices comprised of isotropic, dielectric-only materials. These TO components were shown to be able to achieve efficient light coupling between photonic elements, such as optical sources and waveguides, while exhibiting great design flexibility. Such TO-based GRIN devices, with low losses and broadband operation, have a wide range of applications including optical communications, computing and sensing. Our design approach and examples illustrate the power of the TO methodology to bring GRIN optics into their full potential for advancing practical optical devices and integrated photonic systems.

\section{ACKNOWLEDGMENTS}

Partial support for this work was provided by the NSF MRSEC (Grant No. DMR-0820404). We thank Peter Sieber for his assistance with some of the figure visualizations.

1 Pendry JB, Schurig D, Smith DR. Controlling electromagnetic fields. Science 2006; 312: $1780-1782$.

2 Leonhardt U. Optical conformal mapping. Science 2006; 312: 1777-1780.

3 Schurig D, Mock JJ, Justice BJ, Cummer SA, Pendry JB et al. Metamaterial electromagnetic cloak at microwave frequencies. Science 2006; 314: 977-980.

4 Rahm M, Schurig D, Roberts DA, Cummer SA, Smith DR et al. Design of electromagnetic cloaks and concentrators using form-invariant coordinate transformations of Maxwell's equations. Photon Nanostruct-Fundam Appl 2007; 6: 87-95.

5 Narimanov EE, Kildishev AV. Optical black hole: broadband omnidirectional light absorber. App/ Phys Lett 2009; 95: 041106/1-3.

6 Rahm M, Cummer SA, Schurig D, Pendry JB, Smith DR. Optical design of reflectionless complex media by finite embedded coordinate transformations. Phys Rev Lett 2008; 100: 063903/1-4

7 Rahm M, Roberts DA, Pendry JB, Smith DR. Transformation-optical design of adaptive beam bends and beam expanders. Opt Express 2008; 16: 11555-11567.

8 Kwon DH, Werner DH. Polarization splitter and polarization rotator designs based on transformation optics. Opt Express 2008; 16: 18731-18738.

9 Vakil A, Enghata N. Transformational optics using graphene. Science 2011; 332 : 1291-1294.

10 Kwon DH, Werner DH. Transformation optical designs for wave collimators, flat lenses, and right-angle bends. New J Phys 2008; 10: 115023/1-13.

11 Tang W, Argyropoulos C, Kallos E, Song W, Hao Y. Discrete coordinate transformation for designing all-dielectric flat antennas. IEEE Trans Antenn Propag 2010; 58: 3795-3804.

12 Chen HY, Chan CT, Sheng P. Transformation optics and metamaterials. Nat Mater 2010; 9: 387-396.

13 Kwon DH, Werner DH. Transformation electromagnetics: an overview of the theory and its application. IEEE Antennas Propag Mag 2010; 52: 24-46.

14 Li J, Pendry JB. Hiding under the carpet: a new strategy for cloaking. Phys Rev Lett 2008; 101: 203901/1-4.

15 Liu R, Ji C, Mock JJ, Chin JY, Cui TJ et al. Broadband ground-plane cloak. Science 2009; 323: 366-369.

16 Ma HF, Cui TJ. Three-dimensional broadband ground-plane cloak made of metamaterials. Nat Commun 2010; 1: 1-6.

17 Valentine J, Li J, Zentgraf T, Bartal G, Zhang X. An optical cloak made of dielectrics. Nat Mater 2009; 8: 568-571.

18 Gabrielli LH, Cardenas J, Poitras CB, Lipson M. Silicon nanostructure cloak operating at optical frequencies. Nat Photon 2009; 3: 461-463.

19 Lee J, Blair J, Tamma VA, Wu Q, Rhee SJ et al. Direct visualization of optical frequency invisibility cloak based on silicon nanorod array. Opt Express 2009; 17: 1292212928.
20 Ergin T, Stenger N, Brenner P, Pendry JB, Wegener M. Three-dimensional invisibility cloak at optical wavelengths. Science 2010; 328: 337-339.

21 Kundtz N, Smith DR. Extreme-angle broadband metamaterial lens. Nat Mater 2010; 9: 129-132.

$22 \mathrm{Ma} \mathrm{HF}$, Cui TJ. Three-dimensional broadband and broad-angle transformation-optics lens. Nat Commun 2010; 1: 124/1-7.

23 Zentgraf T, Valentine J, Tapia N, Li J, Zhang X. An optical "Janus" device for integrated photonics. Adv Mater 2010; 22: 2561-2564.

24 Lifante G. Integrated photonics: fundamentals. Chichester: Wiley, 2003.

25 Pollock CR, Lipson M. Integrated photonics. Norwell, MA: Kluwer Academic Publishers, 2003

26 Joannopoulos JD, Villeneuve PR, Fan S. Photonic crystal: put a new twist on light. Nature 1997; 386: 143-149.

27 Vlasov YA, O'Boyle M, Hamann HF, McNab SJ. Active control of slow light on a chip with photonic crystal waveguides. Nature 2005; 438: 65-69.

28 Engheta N. Circuits with light at nanoscales: optical nanocircuits inspired by metamaterials. Science 2007; 317: 1698-1702.

29 Smith DR, Urzhumov Y, Kundtz NB, Landy NI. Enhancing imaging systems using transformation optics. Opt Express 2010; 18: 21238-21251.

30 Thompson JF, Soni BK, Weatherill NP. Handbook of grid generation. Boca Raton, FL: CRC Press, 1999

31 Chang Z, Zhou X, Hu J, Hu G. Design method for quasi-isotropic transformation materials based on inverse Laplace's equation with sliding boundaries. Opt Express 2010; 18: 6089-6096.

32 Landy NI, Padilla WJ. Guiding light with conformal transformations. Opt Express 2009; 17: 14872-14879.

33 Turpin JP, Massoud AT, Jiang ZH, Werner PL, Werner DH. Conformal mappings to achieve simple material parameters for transformation optics devices. Opt Express 2010; 18: 244-252.

34 Tang L, Yin J, Yuan G, Du J, Gao H et al. General conformal transformation method based on Schwarz-Christoffel approach. Opt Express 2011; 19: 15119-15126.

35 Hunt J, Tyler R, Dhar S, Tsai YJ, Bowen P et al. Planar, flattened Luneburg lens at infrared wavelengths. Opt Express 2012; 20: 1706-1713.

36 Zernike F. Luneburg lens for optical waveguide use. Opt Commun 1974; 12: 379 381.

37 Yao SK, Anderson DB, August RR, Youmans BR, Oania CM. Guided-wave optical thinfilm Luneburg lenses: fabrication technique and properties. Appl Opt 1979; 18 4067-4079.

38 Di Falco A, Kehr SC, Leonhardt U. Luneburg lens in silicon photonics. Opt Express 2011; 19: 5156-5162.

39 Gabrielli LH, Lipson M. Integrated Luneburg lens via ultra-strong index gradient on silicon. Opt Express 2011; 19: 20122-20127.

40 Jiang WX, Cui TJ, Ma HF, Zhou XY, Cheng Q. Cylindrical-to-plane-wave conversion via embedded optical transformation. Appl Phys Lett 2008; 92: 261903/1-3.

41 Schmiele M, Varma VS, Rockstuhl C, Lederer F. Designing optical elements from isotropic materials by using transformation optics. Phys Rev A 2010; 81: 033837/ $1-13$.

42 García-Meca C, Martínez A, Leonhardt U. Engineering antenna radiation patterns via quasi-conformal mappings. Opt Express 2011; 19: 23743-23750.

43 Jiang ZH, Gregory MD, Werner DH. Experimental demonstration of a broadband transformation optics lens for highly directive multibeam emission. Phys Rev $B$ 2011; 84: 165111/1-6.

44 Tichit PH, Burokur SN, de Lustrac A. Waveguide taper engineering using coordinate transformation technology. Opt Express 2010; 18: 767-772.

45 Johnson SG, Manolatou C, Fan S, Villeneuve PR, Joannopoulos JD et al. Elimination of cross talk in waveguide intersections. Opt Lett 1998; 23: 1855-1857.

46 Bogaerts W, Dumon P, Thourhout D, Baets R. Low-loss, low cross-talk crossings for silicon-on-insulator nanophotonic waveguides. Opt Lett 2007; 32: 2801-2803.

47 Danner AJ, Tyc T, Leonhardt U. Controlling birefringence in dielectrics. Nat Photon 2011; 5: 357-359.

48 Oulton RF, Sorger VJ, Zentgraf T, Ma RM, Gladden C et al. Plasmon lasers at deep subwavelength scale. Nature 2009; 461: 629-632.

49 Noginov MA, Zhu G, Belgrave AM, Bakker R, Shalaev VM et al. Demonstration of a spaser-based nanolaser. Nature 2009; 460: 1110-1112.

50 Huidobro PA, Nesterov ML, Martin-Moreno L, Garcia-Vidal FJ. Transformation optics for plasmonics. Nano Lett 2010; 10: 1985-1990.

51 Liu Y, Zentgraf T, Bartal G, Zhang X. Transformational plasmon optics. Nano Lett 2010; 10: 1991-1997.

52 Zentgraf T, Liu Y, Mikkelsen MH, Valentine J, Zhang X. Plasmonic Luneburg and Eaton lenses. Nat Nanotechnol 2011; 6: 151-155.

(c) This work is licensed under a Creative Commons Attribution-NonCommercial-NoDerivative Works 3.0 Unported License. To view a copy of this license, visit http:// creativecommons.org/licenses/by-nc-nd/3.0 